\title{
Perturbative charm production and the prompt atmospheric neutrino flux in light of RHIC and LHC
}

\author{
Atri Bhattacharya, ${ }^{a}$ Rikard Enberg, ${ }^{b}$ Mary Hall Reno, ${ }^{c}$ Ina Sarcevic ${ }^{a, d}$ and \\ Anna Stasto ${ }^{e, f}$ \\ ${ }^{a}$ Department of Physics, University of Arizona, \\ 1118 E. Fourth Street, Tucson, AZ, 85721 U.S.A. \\ ${ }^{b}$ Department of Physics and Astronomy, Uppsala University, \\ Box 516, Uppsala, 75120 Sweden \\ ${ }^{c}$ Department of Physics and Astronomy, University of Iowa, \\ 30 North Dubuque Street, Iowa City, IA, 52242 U.S.A. \\ ${ }^{d}$ Department of Astronomy and Steward Observatory, University of Arizona, \\ 933 North Cherry Avenue, Tucson, AZ, 85721 U.S.A. \\ ${ }^{e}$ Department of Physics, Pennsylvania State University, \\ University Park, PA, 16802 U.S.A. \\ ${ }^{f}$ Institute of Nuclear Physics, Polish Academy of Science, \\ ul. Radzikowskiego 152, Kraków, Poland \\ E-mail: atrib@email.arizona.edu, rikard.enberg@physics.uu.se, \\ mary-hall-reno@uiowa.edu, ina@physics.arizona.edu, \\ astasto@phys.psu.edu
}

ABSTRACT: We re-evaluate the prompt atmospheric neutrino flux, using the measured charm cross sections at RHIC and the Large Hadron Collider to constrain perturbative QCD parameters such as the factorization and renormalization scales, as well as modern parton distribution functions and recent estimates of the cosmic-ray spectra. We find that our result for the prompt neutrino flux is lower than previous perturbative QCD estimates and, consequently, alters the signal-to-background statistics of the recent IceCube measurements at high energies.

KEYwords: Neutrino Physics, Solar and Atmospheric Neutrinos, QCD

ArXiv EPrint: 1502.01076 


\section{Contents}

1 Introduction 1

2 Charm production cross section 2

2.1 Differential cross section 5

3 Prompt lepton flux $\quad 6$

$\begin{array}{ll}3.1 \text { Cosmic ray flux } & 7\end{array}$

3.2 Z-moment and prompt lepton flux results 8

3.3 Comparison with previous prompt neutrino flux calculations 11

4 Prompt background at IceCube 12

5 Conclusions 14

\section{Introduction}

Decays of mesons produced when cosmic ray primaries at high energies interact with nuclei in the atmosphere lead to the production of a large flux of neutrinos. The resulting neutrino flux at energies from $10 \mathrm{GeV}-1 \mathrm{TeV}$ has been observed by several pioneering experiments [18] over the last two decades. The dominant contribution to the neutrino flux at these energies comes from the decays of charged pions $\pi^{ \pm} \rightarrow \mu^{ \pm} \nu_{\mu} \rightarrow e^{ \pm} \nu_{e} \nu_{\mu} \nu_{\mu}$, and from leptonic and semi-leptonic decays of kaons [9]. Of late, the construction of first the AMANDA detector [10] and then, its upgrade to the first $\gtrsim 1 \mathrm{~km}^{3}$ volume neutrino telescope, the IceCube (IC) Neutrino Observatory, has made the detection of neutrinos at much higher energies $E_{\nu} \gtrsim 100 \mathrm{TeV}$ possible (see [11] for a review). Moreover, there are plans for the KM3NeT detector in the Mediterranean [12], and for an IceCube-Gen2 upgrade [13], which will reach even higher neutrino energies. While AMANDA had already seen atmospheric neutrinos in the high TeV's [14], IC has recently revealed its first observation of high energy neutrinos with energies going up to $2.1 \mathrm{PeV}[15,16]$. In total, IC observes 37 events in the energy range $10 \mathrm{TeV}-2.1 \mathrm{PeV}$ over 988 days, with atmospheric neutrinos and muons expected to be responsible for about $10-11$ of those, their fluxes being especially significant for energies up to $200 \mathrm{TeV}$. A precise understanding of the prompt neutrino background at these energies is crucial in determining the IC signal statistics. Because present values for the flux used by IC depend on theoretical results ${ }^{1}$ [18-22], it is timely to revisit the theoretical predictions in light of updated information on the various QCD inputs as well as recent high-energy experimental results such as those obtained at the Large Hadron Collider (LHC).

Prompt neutrinos, produced in the atmosphere by decays of charmed hadrons (e.g., $\left.D^{0}, D^{ \pm} \rightarrow \nu_{e, \mu} X\right)$ that come from cosmic-ray interactions with atmospheric nuclei $(p N \rightarrow$

\footnotetext{
${ }^{1}$ IceCube has recently published an upper limit [17] on the prompt flux at energies above $1 \mathrm{TeV}$ at less than 1.52 times the flux predicted in ref. [18].
} 
$c \bar{c} X)$, become an important component of the atmospheric neutrino flux at these high energies [18-22]. For the IC study of neutrinos from astrophysical sources, atmospheric neutrinos are a background and therefore, need to be carefully estimated. The neutrino flux from pions and kaons, hereafter called the conventional neutrino flux, has been previously calibrated against multi-GeV atmospheric neutrino observations. This conventional flux is well understood, but the prompt neutrino flux, being sub-dominant at lower energies, is less so.

The evaluation of the prompt flux requires as a first step the computation of the charm production cross section in the process $p N \rightarrow c \bar{c} X$, followed by the hadronization of charm particles. The computation of charm production in hadronic collisions introduces some of the biggest uncertainties that translate into the evaluation of the prompt flux. These arise due to uncertainties in the knowledge of a) the charm mass $\left.\left(m_{\mathrm{c}}\right), b\right)$ the factorization $\left(M_{\mathrm{F}}\right)$ and renormalization $\left(\mu_{\mathrm{R}}\right)$ scales, and $\left.c\right)$ the choice of parton distribution functions (PDF's). In addition, the final step in computing the prompt lepton flux involves folding the charmed hadron production cross section with the flux of incoming cosmic-rays, and thus incurs large uncertainties from the limited understanding of the extremely high-energy cosmic ray composition.

Two approaches to the calculation of the prompt lepton flux are via perturbative QCD (pQCD) in the parton model (as in, e.g., [20-22]) and with the dipole model (see, e.g., [18]). Recently, the production of charm mesons in the atmosphere has also been studied using the event generator SIBYLL [23]. In this paper we focus on a new evaluation of the prompt flux using next-to-leading order (NLO) QCD. We use recently updated PDF's in our pQCD calculations while limiting the uncertainties in the charm production computation by incorporating constraints from related high energy collider results. Finally, we evaluate the prompt neutrino flux and, therefore, the prompt atmospheric neutrino background for IC by using recent estimates of the cosmic-ray spectrum and composition [24-26] and our updated charm production cross section.

Our focus here is on the link between the charm cross section measurements, including LHC results, and the prompt neutrino flux in the context of pQCD. This allows us to make estimates of some of the uncertainties in the flux evaluation. This work is part of a broader program to assess the uncertainties in the theoretical evaluation of the prompt flux accounting for a wider range of approaches to calculating the charmed hadron cross sections [27].

In the next section, we review the pQCD evaluation of the production of charmed hadrons. In section 3 , we describe the $Z$-moment approach to evaluation the flux, and we show our results for the prompt muon (and electron) neutrino plus antineutrino fluxes. We briefly discuss the implications of this new flux for the background evaluation at IceCube in section 4 , before drawing our conclusions in the final section.

\section{Charm production cross section}

The PeV energy range for atmospheric neutrinos corresponds to an incident energy $E_{p} \sim 30$ $\mathrm{PeV}$ for $p A$ fixed target interactions. The LHC center of mass energy $\sqrt{s}=7 \mathrm{TeV}$ is 


\begin{tabular}{|c|c|c|}
\hline Expt. & $\sqrt{s}[\mathrm{TeV}]$ & $\sigma[\mathrm{mb}]$ \\
\hline PHENIX [31] & 0.20 & $\mathbf{0 . 5 5 1} \mathbf{1}_{-0.231}^{+0.203}($ sys $)$ \\
\hline STAR [32] & 0.20 & $\mathbf{0 . 7 9 7} \pm 0.210(\text { stat })_{-0.295}^{+0.208}$ (sys) \\
\hline ALICE [28] & 2.76 & $\begin{array}{c}4.8 \pm 0.8(\text { stat })_{-1.3}^{+1.0}(\text { sys }) \pm 0.06(\mathrm{BR}) \\
\pm 0.1(\text { frag }) \pm 0.1(\text { lum })_{-0.4}^{+2.6}(\text { extrap })\end{array}$ \\
\hline ALICE [28] & 7.00 & $\begin{array}{c}8.5 \pm 0.5(\text { stat })_{-2.4}^{+1.0}(\mathrm{sys}) \pm 0.1(\mathrm{BR}) \\
\pm 0.2(\mathrm{frag}) \pm 0.3(\mathrm{lum})_{-0.4}^{+5.0}(\text { extrap })\end{array}$ \\
\hline ATLAS [29] & 7.00 & $\begin{array}{c}7.13 \pm 0.28(\text { stat })_{-0.66}^{+0.90}(\text { sys }) \\
\pm 0.78(\text { lum })_{-1.90}^{+3.82}(\text { extrap })\end{array}$ \\
\hline $\mathrm{LHCb}[30]$ & 7.00 & $\mathbf{6 . 1 0 0} \pm 0.930$ \\
\hline
\end{tabular}

Table 1. Total cross section for $p p(p N) \rightarrow c \bar{c} X$ in hadronic collisions, extrapolated based on NLO QCD by the experimental collaborations from charmed hadron production measurements in a limited phase space region.

equivalent to a fixed target beam energy in $p p$ collisions of $E_{\mathrm{b}}=26 \mathrm{PeV}$. The LHC measurements of the charm production cross section [28-30] together with recent RHIC [31, 32 ] and modern parton distribution functions (PDFs) have narrowed down some of the uncertainty in the rate of charm production in the atmosphere. The experimental results at high energy for the charm production cross section in hadronic collisions are listed in table 1.

In ref. [33], Nelson, Vogt and Frawley have investigated a range of factorization and renormalization scales using the CT10 PDF's [34] and the NLO order QCD calculation of Nason, Dawson and Ellis [35, 36]. Using a charm quark mass central value of $m_{\mathrm{c}}=1.27 \mathrm{GeV}$ based on lattice QCD determinations of the charm quark mass, as summarized in ref. [37], and a combination of fixed target, PHENIX, and STAR charm production cross sections, they find that $M_{\mathrm{F}} / m_{\mathrm{c}}=1.3-4.3$ and $\mu_{\mathrm{R}} / m_{\mathrm{c}}=1.7-1.5$ with $M_{\mathrm{F}}=2.1 m_{\mathrm{c}}$ and $\mu_{\mathrm{R}}=1.6 m_{\mathrm{c}}$ as central values. We use these values of parameters as a guide to the range of theoretical NLO charm cross sections expected at high energies.

In our calculation we use the NLO Fortran code of Cacciari et al. [38, 39] that includes the total cross section [35] as well as the single [36] and double differential [40] distributions of charm (i.e., $\mathrm{d} \sigma / \mathrm{d} y$ and $\mathrm{d}^{2} \sigma / \mathrm{d} y \mathrm{~d} p_{\mathrm{T}}$ respectively). The cross sections shown in figure 1 for proton interactions with the iso-scalar nucleon ${ }^{2} p N \rightarrow c \bar{c} X$ are obtained using factorization and renormalization scales relative to the charmed quark transverse mass $m_{\mathrm{T}}^{2} \equiv\left(m_{\mathrm{c}}^{2}+p_{\mathrm{T}}^{2}\right)$ rather than relative to the charm quark mass $m_{\mathrm{c}}$. This choice of the scale dependence is an acceptable representation of the factorization and renormalization scales both at moderate energies (where the choice of scale is not sensitive to $p_{\mathrm{T}}$ ) and at high energies (where the

\footnotetext{
${ }^{2}$ Since the charm production cross section is dominated by gluon fusion, $p \simeq n \simeq N$.
} 


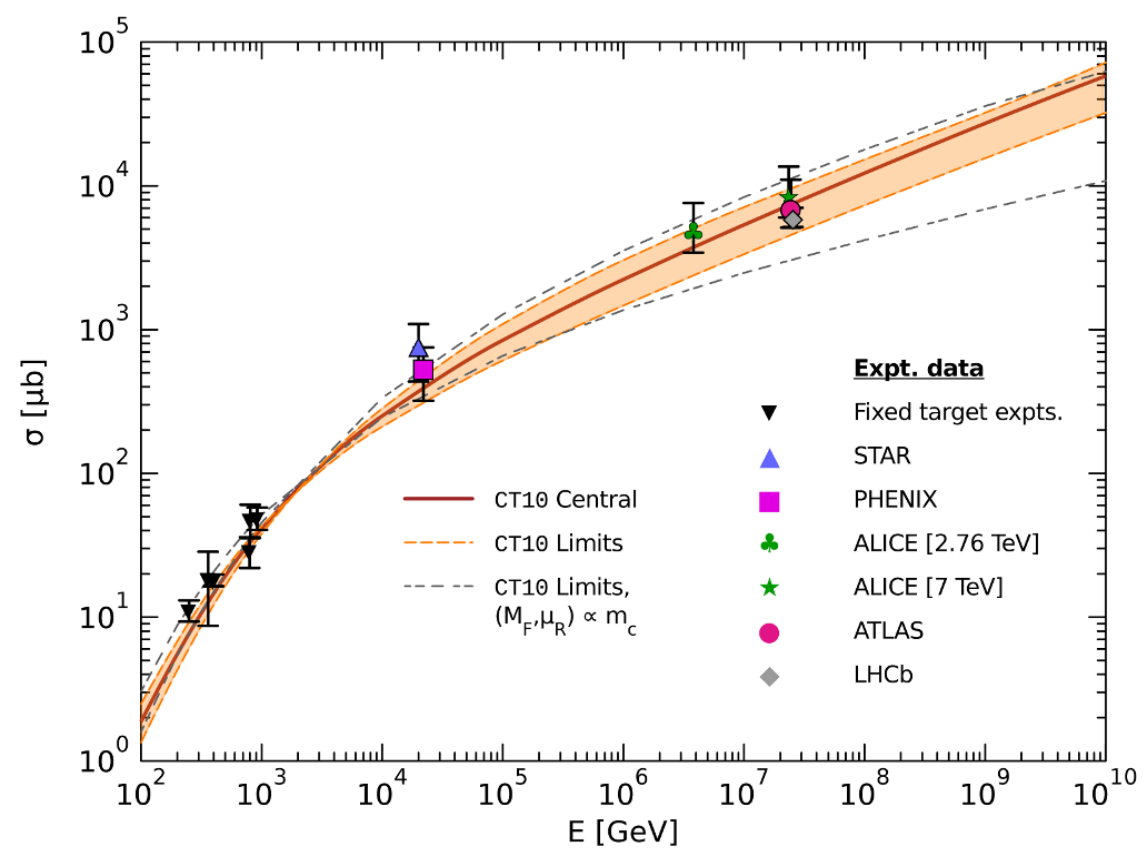

Figure 1. The charm production cross section $\sigma_{p N \rightarrow c \bar{c}+X}$ at NLO with $m_{\mathrm{c}}=1.27 \mathrm{GeV}$ using the CT10 parton distributions for a range of scales described in the text, with the central set with factorization and renormalization scales $M_{\mathrm{F}}=2.10 m_{\mathrm{T}}$ and $\mu_{\mathrm{R}}=1.6 m_{\mathrm{T}}$, respectively. Apart from experimental data points listed in table 1, results from HERA-B [43] and lower energy experiments summarized in [44] for $p N$ scattering are shown (labelled as Fixed target expts.). For comparison, we also show the lower and upper limits (grey fine-dashed curves) when the renormalization and factorization scales are made to vary proportionally to $m_{\mathrm{c}}$ rather than to $m_{\mathrm{T}}$.

$p_{\mathrm{T}}$ dependence in the scales becomes important). The CT10 PDFs are our default choice for the NLO evaluations of charm production. The shaded band in figure 1 comes from using $\left(M_{\mathrm{F}}, \mu_{\mathrm{R}}\right)=(1.25,1.48) m_{\mathrm{T}}$ and $\left(M_{\mathrm{F}}, \mu_{\mathrm{R}}\right)=(4.65,1.71) m_{\mathrm{T}}$ (labeled as CT10 Limits), as suggested in ref. [33]. The central solid curve (CT10 Central) is obtained using $\left(M_{\mathrm{F}}, \mu_{\mathrm{R}}\right)=(2.1,1.6) m_{\mathrm{T}}$. The LHCb [30], ALICE [28] and ATLAS [29] cross sections measurements are within our calculated cross section uncertainty band, thus we use this range of scales for our flux uncertainty band. The range of QCD parameters consistent with observations have been studied in [33] by taking into consideration both total as well as differential cross section measurements at the LHC for a wide range of the kinematic phase space. This includes measurements at low transverse momenta and forward rapidities. The upper and lower limits of our QCD calculation, consistent to within the error-bars for the experimental data, are based on these parameter uncertainties (see e.g., shaded area in figure 1).

The total $p p \rightarrow c \bar{c} X$ cross section at high energies is dominated by gluon fusion, so the cross section is sensitive to the gluon PDF small- $x$ behavior. The perturbative results in [21] overestimate the charm cross section because of the steeply rising gluon PDFs at small- $x$ for the CTEQ3 PDF sets [41] used, e.g., in [18, 21]. By comparison, the updated CT10 PDFs we use here have a more slowly growing behavior as $x$ decreases. These PDFs are fit based on recent experimental inputs (including from LHC, Tevatron and others). 
Using the MSTW [42] PDFs, which have an even smaller gluon PDF growth at small- $x$, leads to cross sections that grow even more slowly (compared to CT10) at higher energies, however, the results still fall within the theoretical uncertainty band shown in figure 1 .

By choosing $M_{\mathrm{F}}$ and $\mu_{\mathrm{R}}$ with $m_{\mathrm{T}} \rightarrow m_{\mathrm{c}}$, we find a similar energy dependence of the total charm cross section [33]. In addition, the central values for the charm cross sections are roughly comparable for the same choice of PDF. Using the CT10 PDF and $m_{\mathrm{c}}=1.27 \mathrm{GeV}$, in the energy range $E_{\mathrm{b}}=10^{6}-10^{8} \mathrm{GeV}$, the cross section obtained with $\left(M_{\mathrm{F}}, \mu_{\mathrm{R}}\right)=(2.1,1.6) m_{\mathrm{c}}$ is within about $\pm 10 \%$ of the cross section calculated with $\left(M_{\mathrm{F}}, \mu_{\mathrm{R}}\right)=(2.1,1.6) m_{\mathrm{T}}$. The upper choice of scale factors multiplying $m_{\mathrm{c}}$ gives a comparable cross section for the same factors multiplying $m_{\mathrm{T}}$. For the lower range of scale factors, the high energy charm pair production cross section is smaller for $m_{\mathrm{c}}$ dependent scales. For example, for $E_{\mathrm{b}}=10^{8} \mathrm{GeV}$ using $\left(M_{\mathrm{F}}, \mu_{\mathrm{R}}\right)=(1.25,1.48) m_{\mathrm{c}}$, the cross section is about $40 \%$ lower than the cross section evaluated using $m_{\mathrm{T}}$ dependent scales as shown by the grey fine-dashed curves in figure 1. Consequently, while the central values for either choice of the scale give almost identical prompt neutrino flux, it is the uncertainty band that is smaller for the $\sim m_{\mathrm{T}}$ scale choice.

As discussed below, we use the superposition approximation in describing the cosmic ray interactions with air nuclei [45]. In this approximation, the cosmic ray all-particle flux is reduced to a nucleon flux. We require the $N A \rightarrow c \bar{c} X$ cross section, and use $p \simeq n \simeq N$ (as noted previously). We continue to label the cosmic ray nucleon with $p$, to distinguish it as the beam nucleon as opposed to the target nucleon. The average atomic number of the target air nuclei is $A=14.5$. Measurements of charm production on a variety of targets [46, 47] show that $\sigma(p A \rightarrow c \bar{c} X) \simeq A \sigma(p N \rightarrow c \bar{c} X)$. We use this approximation here.

\subsection{Differential cross section}

While we seek compatibility of the total charm quark pair production cross section with the results reported by the experimental collaborations, the dominant contribution to the prompt flux is from forward production of charm, including fragmentation into charmed hadrons. In our semi-analytic evaluation of the prompt atmospheric lepton flux, we require the differential charmed hadron energy distribution,

$$
\frac{d \sigma}{d E_{h}}=\sum_{k} \int \frac{d \sigma}{d E_{k}}(A B \rightarrow k X) D_{k}^{h}\left(\frac{E_{h}}{E_{k}}\right) \frac{d E_{k}}{E_{k}}
$$

in terms of the parton level differential distribution and the fragmentation function $D_{k}^{h}$. Here, $h=D^{ \pm}, D^{0}\left(\bar{D}^{0}\right), D_{s}^{ \pm}, \Lambda_{c}^{ \pm}$and $k=c, \bar{c}$. We approximate the fragmentation functions for charmed hadrons as energy independent. Eq. (2.1) can be written as

$$
\frac{d \sigma}{d x_{E}}(p A \rightarrow h X)=A \int_{x_{E}}^{1} \frac{d z}{z} \frac{d \sigma}{d x_{c}}(p N \rightarrow c X) D_{c}^{h}(z)
$$

in terms of $x_{E}=E_{h} / E_{\mathrm{b}}$ and $x_{c}=E_{c} / E_{\mathrm{b}}=x_{E} / z$ for an incident cosmic ray nucleon energy (beam energy) $E_{\mathrm{b}}$. In figure 2, we show the differential cross section as a function of $x_{c}$ 


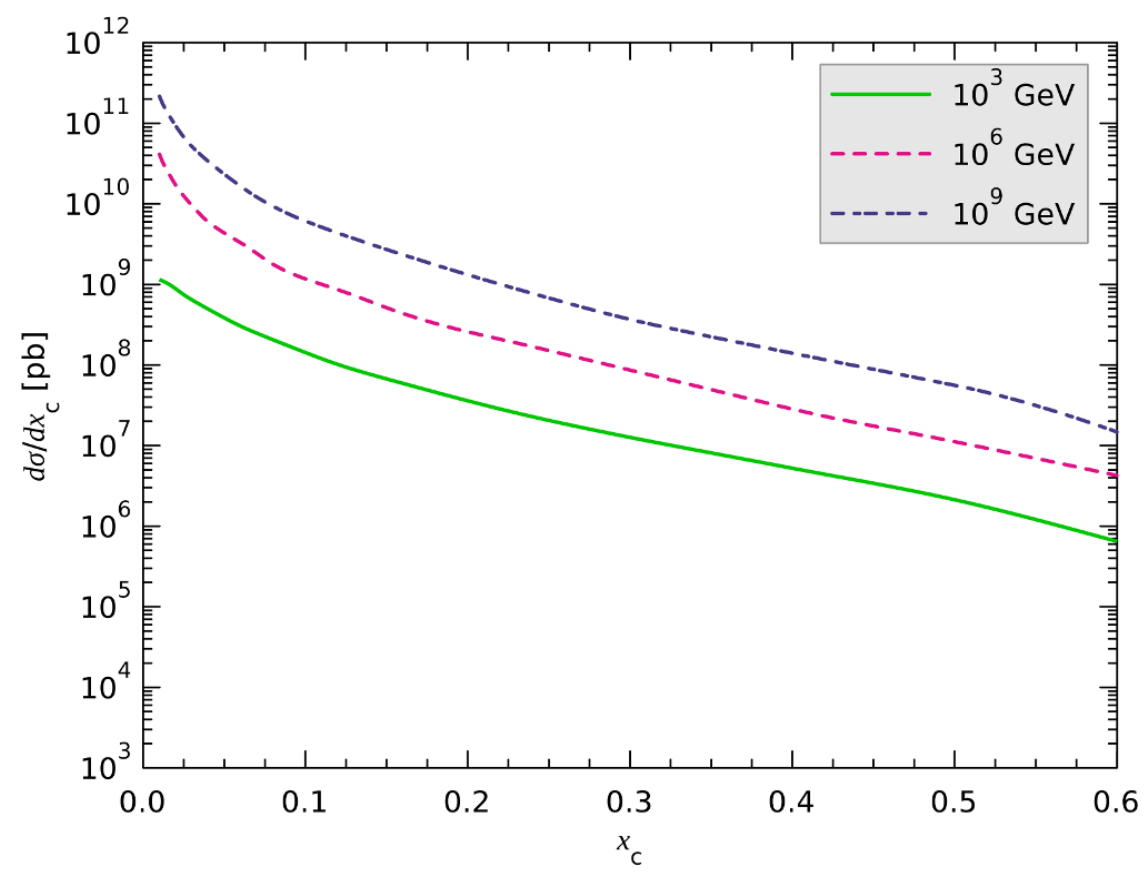

Figure 2. The differential cross section $d \sigma / d x_{c}$ for the charmed quark, as a function of $x_{c}$ for $E=10^{3}, 10^{6}, 10^{9} \mathrm{GeV}$ for $m_{\mathrm{c}}=1.27 \mathrm{GeV}$ and $M_{\mathrm{F}}=2.1 m_{\mathrm{T}}, \mu_{\mathrm{R}}=1.6 m_{\mathrm{T}}$ using the CT10 NLO PDFs.

for $E_{\mathrm{b}}=10^{3}, 10^{6}$ and $10^{9} \mathrm{GeV}$ in $p N$ scattering, here for $\left(M_{\mathrm{F}}, \mu_{\mathrm{R}}\right)=(2.1,1.6) m_{\mathrm{T}}$. The distributions for $\left(M_{\mathrm{F}}, \mu_{\mathrm{R}}\right)=(2.1,1.6) m_{\mathrm{c}}$ are very similar.

We can compare our results here to those obtained previously, notably in [21]. With the CT10 NLO PDF's, the $m_{\mathrm{T}}$ dependent scales, and a full NLO calculation, we find that our differential distribution at low $x$ is lower than in ref. [21] at high energies (e.g., about $28 \%$ lower at $10^{9} \mathrm{GeV}$ for $x=0.1$ ). As previously discussed, this stems from the use of updated PDFs which have a slower growth at small $x$ than the CTEQ3 PDFs used in [21].

Our default choice of fragmentation functions for charmed hadrons is that of Kniehl and Kramer [48]. The net effect of including the fragmentation functions is to reduce the predicted flux by about $\sim 30 \%$ relative to the flux without fragmentation included.

\section{Prompt lepton flux}

We use the $Z$-moment approach $[49,50]$, including an energy dependence of the $Z$-moments [20] and approximating the depth of the atmosphere as infinite. In the exponential atmosphere approximation where the density is

$$
\rho(h)=\rho_{0} \exp \left(-h / h_{0}\right)
$$

for $\rho_{0}=2.03 \times 10^{-3} \mathrm{~g} / \mathrm{cm}^{3}$ and $h_{0}=6.4 \mathrm{~km}$, the low energy and high energy lepton fluxes have particularly simple forms, involving the spectrum weighted $Z$-moments. The production moments are defined by

$$
Z_{p h}\left(E_{h}\right)=\int_{x_{E_{\min }}}^{1} \frac{d x_{E}}{x_{E}} \frac{\phi_{p}^{0}\left(E_{h} / x_{E}\right)}{\phi_{p}^{0}\left(E_{h}\right)} \frac{1}{\sigma_{p A}\left(E_{h}\right)} \times A \frac{d \sigma}{d x_{E}}(p N \rightarrow h X) .
$$


The all-nucleon cosmic ray flux as a function of atmospheric column depth $X$ is

$$
\phi_{p}(E, X) \simeq \phi_{p}^{0}(E) \exp \left(-X / \Lambda_{p}\right)=(d N / d E) \exp \left(-X / \Lambda_{p}\right),
$$

with $\Lambda_{p}=\lambda_{p}(E) /\left(1-Z_{p p}(E)\right)$. For the proton-air cross section, we use an approximate parametrization of the EPOS 1.99 cross section [51] that is consistent with the high energy results of the Pierre Auger Observatory [52].

For decays, the differential cross section is replaced by the differential decay distribution, and the cosmic ray flux is replaced by the charmed hadron flux. At high energies, we evaluate the high energy decay $Z$-moment with a spectral weight of $\phi_{p}^{0}\left(E / x_{E}\right) / \phi_{p}^{0}(E)$ since $\phi_{h} \propto \phi_{p}$ at high energies. The low energy decay $Z$-moment is evaluated using a spectral weight of $1 / x_{E} \cdot \phi_{p}^{0}\left(E / x_{E}\right) / \phi_{p}^{0}(E)$ since the low energy charmed hadron flux is proportional to $E \phi_{p}^{0}(E)$.

For low energy, the lepton $\ell=\mu, \nu_{i}$ flux from $h \rightarrow \ell$ decays is approximated by

$$
\phi_{\ell}^{\text {low }}(h)=Z_{h \ell}^{\text {low }} \frac{Z_{p h}}{1-Z_{p p}} \phi_{p}^{0},
$$

while for high energy (see e.g. [20]),

$$
\phi_{\ell}^{\text {high }}(h)=Z_{h \ell}^{\text {high }} \frac{Z_{p h}}{1-Z_{p p}} \frac{\ln \left(\Lambda_{h} / \Lambda_{p}\right)}{1-\Lambda_{p} / \Lambda_{h}} \phi_{p}^{0} .
$$

Each $Z$ factor, $\Lambda$ and $\phi_{p}^{0}$ has an energy dependence, suppressed in the notation above. The resulting lepton flux from charmed hadrons $h$ is

$$
\phi_{\ell}=\sum_{h} \frac{\phi_{\ell}^{\text {low }}(h) \phi_{\ell}^{\text {high }}(h)}{\phi_{\ell}^{\text {low }}(h)+\phi_{\ell}^{\text {high }}(h)} .
$$

The lepton fluxes from atmospheric charm depend on the cosmic ray flux directly through $\phi_{p}^{0}(E)$ and in the evaluation of the energy dependent $Z$-moments.

\subsection{Cosmic ray flux}

The cosmic ray flux has been measured directly and indirectly over a wide energy range. Direct measurements are available to energies of about $\sim 100 \mathrm{TeV}$. At higher energies, indirect measurements are made by air shower array experiments. While there are some discrepancies in the normalization of the cosmic ray spectrum at high energies, overall the all-particle cosmic ray spectrum for the energy range of interest, $10^{3}-10^{10} \mathrm{GeV}$, approximately follows a broken power-law with the break occurring at $E \simeq 5 \times 10^{6} \mathrm{GeV}$. Many earlier evaluations of the prompt lepton flux [18, 20-22, 53] used the broken power-law form for the nucleon flux with [20]:

$$
\phi_{p}^{0}(E)= \begin{cases}1.7 E^{-2.7} & \text { for } \quad E<5 \cdot 10^{6} \mathrm{GeV} \\ 174 E^{-3} & \text { for } \quad E>5 \cdot 10^{6} \mathrm{GeV},\end{cases}
$$

for $E$ in $\mathrm{GeV}$ and the nucleon flux in units of $\mathrm{cm}^{-2} \mathrm{~s}^{-1} \mathrm{sr}^{-1} \mathrm{GeV}^{-1}$. With the fairly recent measurements from ATIC [54], CREAM [55, 56] and Pamela [57], combined with earlier 


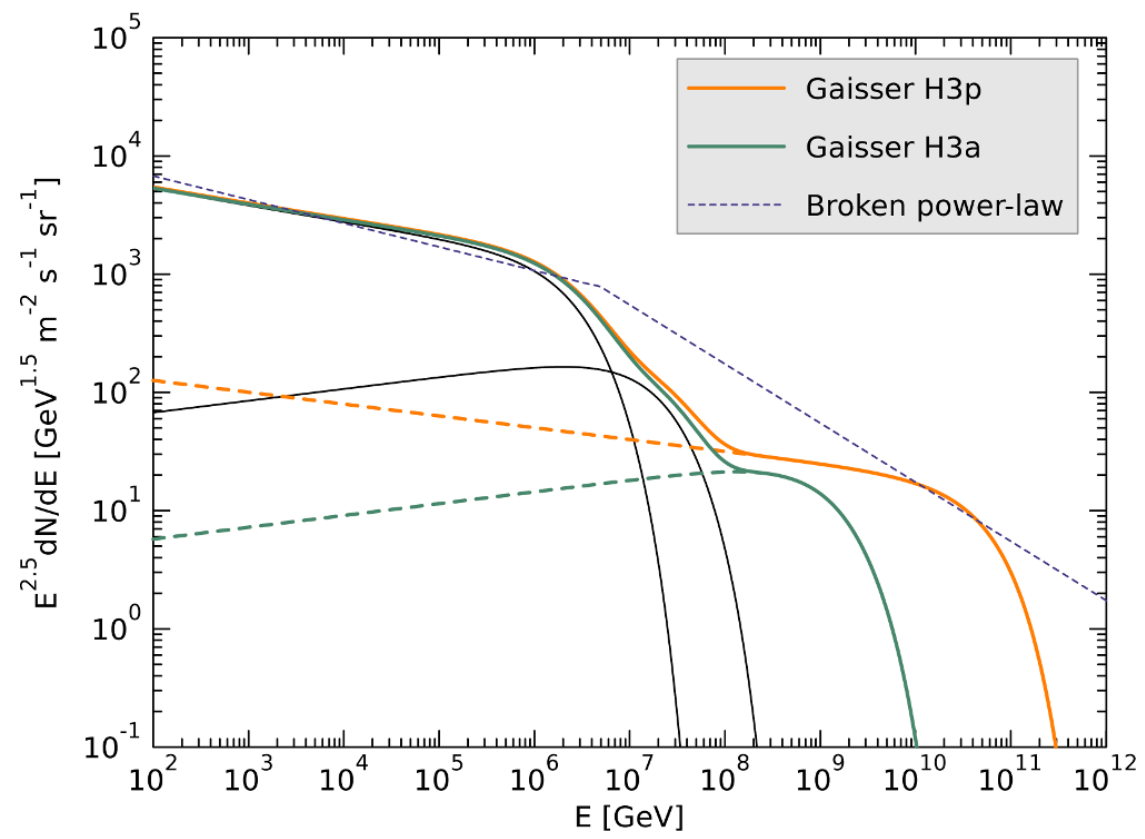

Figure 3. The all-nucleon cosmic ray spectrum as a function of energy per nucleon for the three component model of ref. [24] with a mixed extragalactic population (H3a) and all proton extragalactic population (H3p), and for the broken power-law of eq. (3.7).

measurements, Gaisser [24] and collaborators [25, 26] have taken a multicomponent model with three or four source populations to develop models for the cosmic ray composition. Their parametrizations depend on the particles' electric charges $Z$ and maximum energies of the source populations, with spectral indices $\gamma$ that vary by population and nucleus. We use here the parametrization by Gaisser in ref. [24] with three populations: from supernova remnants, from other galactic sources and from extragalactic sources. The H3a flux from ref. [24] has a mixed composition in the extragalactic population, while the extragalactic population in what we call the H3p flux is all protons. Thus, the cosmic ray nucleon spectrum is identical for H3a and H3p for nucleon energies below $\sim 10^{7} \mathrm{GeV}$. Converting the all-particle flux to the nucleon flux, the H3a and H3p fluxes are shown along with the broken power-law in figure 3 .

The composition of the cosmic rays causes a much steeper drop in the nucleon flux above the knee energy than when using the simple broken power-law parametrization. This is particularly important for the high energy prompt lepton flux. To allow for comparisons with earlier work, we show our results for the prompt lepton flux for the broken power-law and the H3a and H3p cosmic ray fluxes.

\section{2 $Z$-moment and prompt lepton flux results}

The production $Z$-moments are shown as a function of energy in figure 4 a. For each of $Z_{p D^{0}}$ and $Z_{p D^{ \pm}}$, the three curves show the moments evaluated for the three respective cosmic-ray nucleon fluxes presented in figure 3 . For the H3p flux, we also show the band of $Z$-moments from the range of differential cross sections by taking $\left(M_{\mathrm{F}}, \mu_{\mathrm{R}}\right)=(1.25,1.48) m_{\mathrm{T}}$ (for lower limit) and $\left(M_{\mathrm{F}}, \mu_{\mathrm{R}}\right)=(4.65,1.71) m_{\mathrm{T}}$ (for upper limit). This relative band of variation 


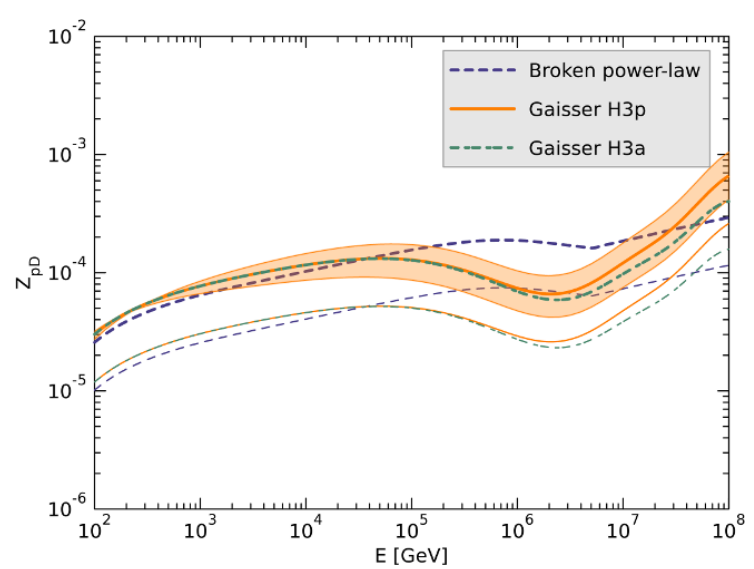

(a)

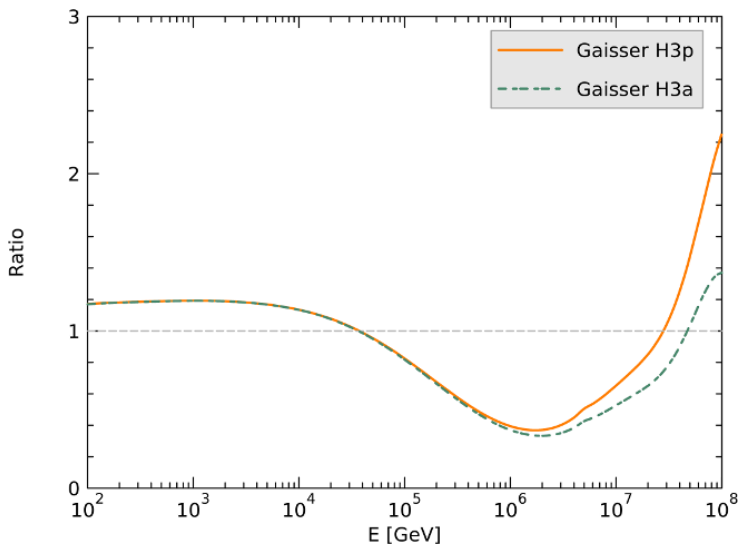

(b)

Figure 4. (a) Production $Z$-moments for $p A \rightarrow M$ for $M=D^{0}+\bar{D}^{0}$ (as thick curves) and $M=D^{ \pm}$ (thin curves) for H3a (green dot-dashed curves), H3p (orange solid curves) and broken power-law (blue short-dashed curves) cosmic ray fluxes. The range of variation for the $Z$-moments is shown (orange shaded region) for the $Z_{p D^{0}}$ when the H3p cosmic ray flux is used for computation, and the relative range of variation is identical for the other curves.

(b) Ratio of central $Z$-moments for $p A \rightarrow D^{0}+\bar{D}^{0}$ using the Gaisser H3a and H3p fluxes to the broken power-law cosmic ray flux (from eq. (3.7)).

is identical for the other $Z$-moments shown in the figure. Figure $4 \mathrm{~b}$ shows the ratio of the

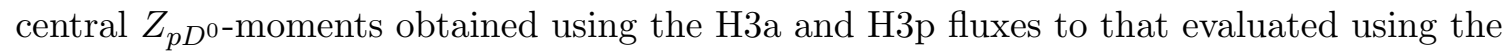
broken power-law nucleon flux.

The major difference between the $D$-meson production $Z$-moments when using the power-law CR flux [from eq. (3.7)] against a more recent CR flux estimate, such as the Gaisser H3p flux, arises at the high energies $\geqslant 10^{5} \mathrm{GeV}$, where the latter noticeably dip, before rising sharply at energies beyond the tens of $\mathrm{PeV}$. In contrast, the $Z$-moments when using the broken power-law follows a more steady behavior. This difference in nature can be traced to the particular behavior of the Gaisser cosmic ray primary fluxes - a significant softening of the spectral shape occurs at around a few $\mathrm{PeV}$ energies, where the population transitions from being dominantly galactic to extra-galactic, before the spectra hardens again at energies around a few hundred $\mathrm{PeV}$ (see figure 3). When translated to the production $Z$-moments, these effects are visible at comparatively lower energies because of the inelasticity of the high energy $p p$ collision, which implies that only a small fraction (given by $\left\langle x_{E}\right\rangle \approx 0.1$ ) of the incident proton energy goes into the produced $c \bar{c}$. The nature of the $Z$-moments, in turn, translates directly to the total prompt lepton flux (as shown in figure $5 \mathrm{a}$ ). The central $Z$-moments obtained using the H3p estimate will henceforth be our benchmark result when determining the prompt flux and correspondingly the event-rates at IC.

As discussed above, we use the charmed hadron spectral weights for the decay $Z$ moments. These are evaluated using $d n / d E$ from ref. [49, 50, 58].

Additional $Z$-moments are needed for the flux evaluation, in particular $Z_{p p}$ and $Z_{h h}$ along with $\lambda_{h}$. For $Z_{p p}$, we have approximated the $p A \rightarrow p X$ differential cross section with 


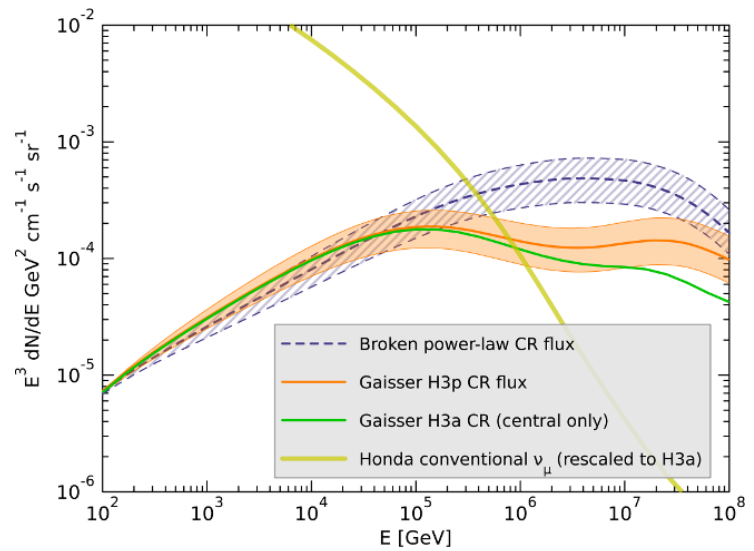

(a)

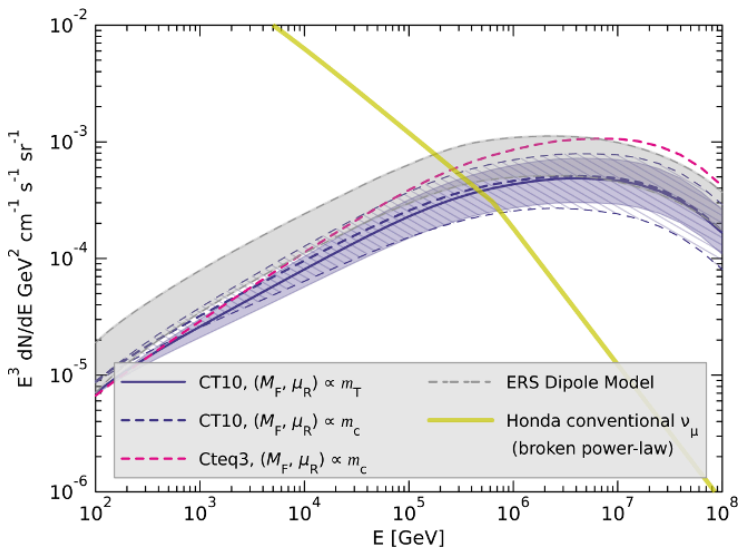

(b)

Figure 5. (a) Our benchmark results for the prompt $\nu_{\mu}+\bar{\nu}_{\mu}$ flux scaled by $E^{3}$ is shown as an orange curve, with the cosmic ray flux given by the Gaisser H3p fit (see figure 3). The blue curve uses instead a broken power-law (as used in previous analyses, e.g., [18]). For each curve, the associated shaded region indicates the uncertainty due to variation of the QCD parameters. The vertical conventional flux from Honda (see, e.g., [20]), reweighted to the H3a cosmic-ray primary flux, is also shown.

(b) Comparison of neutrino fluxes with variation in scales and PDFs for the broken power-law CR primary flux. Shown are the results for central values obtained using CT10 as the PDF with $\left(M_{\mathrm{F}}, \mu_{\mathrm{R}}\right) \propto m_{\mathrm{T}}$ (solid slate blue line) and with scales $\left(M_{\mathrm{F}}, \mu_{\mathrm{R}}\right) \propto m_{\mathrm{c}}$ (dashed slate blue curve) along with their associated bands of variation (corresponding to QCD parameters discussed in text) as solid and hatched fills for scales proportional to $m_{\mathrm{T}}$ and $m_{\mathrm{c}}$ respectively. The central flux (corresponding to $M_{\mathrm{F}}=2.1 m_{\mathrm{c}}, \mu_{\mathrm{R}}=1.6 m_{\mathrm{T}}$ ) evaluated for CTEQ3 as the PDF is shown as the pink dashed curve, along with the dipole model computation (gray short-dashed curve) of ref. [18]. The flux uncertainty from [18] is shown as a grey band. For comparison, the vertical conventional flux from Honda (see, e.g., [20]), based on the broken power-law cosmic-ray primary flux, is also shown.

a scaling form

$$
\frac{d \sigma}{d x_{E}} \simeq \sigma_{p A}(E)(1+n)\left(1-x_{E}\right)^{n}
$$

with $\sigma_{p A}$ as described above and $n=0.51$. With these choices, at $E=10^{3} \mathrm{GeV}$ for the broken power-law, $Z_{p p}=0.271$ and $\Lambda_{p}=\lambda_{p} /\left(1-Z_{p p}\right)=116 \mathrm{~g} / \mathrm{cm}^{2}$. By comparison, the scaling values in $[49,50]$ are $Z_{p p}=0.263$ and $\Lambda_{p}=117 \mathrm{~g} / \mathrm{cm}^{2}$. The change in the cosmic ray spectrum with the broken power-law together with the energy dependence of the cross section reduces our calculated $Z_{p p}$ to 0.231 and $\Lambda_{p}$ to $67 \mathrm{~g} / \mathrm{cm}^{2}$ at $E=10^{8} \mathrm{GeV}$. Similar results are obtained for the H3a and H3p cosmic ray flux inputs. We remark that in [20], energy dependent $Z$-moments evaluated using the PYTHIA Monte Carlo program [59] are used, giving e.g. $Z_{p p}\left(10^{3} \mathrm{GeV}\right) \simeq 0.5$. These are also used in [18]. The low energy flux is proportional to $\left(1-Z_{p p}\right)^{-1}$, so this numerical factor is important to the overall normalization.

For the charmed hadrons' interaction lengths and interaction $Z$-moments, we use kaonproton interactions as representative. For all charmed hadrons, we use the same expres- 
sions, based on kaons. We take

$$
\frac{d \sigma}{d x_{E}} \simeq A^{0.75} \sigma_{K N}(E)(1+n)\left(1-x_{E}\right)^{n}
$$

with $\sigma_{K N}$ determined by the COMPAS group and summarized by the Particle Data Group in [37]. We find that setting $n=1$ gives $Z_{K K}=0.217$ and $\Lambda_{K}=162 \mathrm{~g} / \mathrm{cm}^{2}$ for the broken power-law at $10^{3} \mathrm{GeV}$, reducing to $Z_{K K}=0.176$ and $\Lambda_{K}=40 \mathrm{~g} / \mathrm{cm}^{2}$ at $10^{8} \mathrm{GeV}$. The scaling values in $[49,50]$ are 0.211 and $175 \mathrm{~g} / \mathrm{cm}^{2}$, respectively. The precise value of $n$ for meson scattering in eq. (3.9) affects only $\phi_{\ell}^{\text {high }}$.

Our prompt lepton fluxes are shown in figure 5a. We show $E^{3} \phi_{\nu_{\mu}+\bar{\nu}_{\mu}}$ as a function of neutrino energy. The fluxes of $\mu+\bar{\mu}$ and $\nu_{e}+\bar{\nu}_{e}$ are the same as shown in the figure, in the approximations we use here. The upper band shows our NLO result using CT10 PDFs with the range of $\left(M_{\mathrm{F}}, \mu_{\mathrm{R}}\right)$ discussed in section 2, using the broken power-law as the input cosmic ray all-nucleon spectrum. The lower band shows the prompt flux using the H3p cosmic ray flux inputs. The H3a cosmic ray flux results in a lower prompt lepton flux for energies above $\sim 2 \times 10^{5} \mathrm{GeV}$, roughly a factor of two lower at $E=10^{8} \mathrm{GeV}$.

\subsection{Comparison with previous prompt neutrino flux calculations}

In [18], the dipole model approach including effects of parton saturation was used for charm production in $p p \rightarrow c \bar{c} X$. With respect to the total neutrino flux obtained therein, we find that our benchmark results, i.e., obtained using Gaisser H3p cosmic ray flux as opposed to the use of broken power-law spectra in the former, yields prompt neutrino fluxes that are reduced by a factor which varies from about 2 at lower energies (below $100 \mathrm{TeV}$ ) to a maximum reduction factor of about 8 at high energies (few PeV). On the contrary, the result for the central value of the flux obtained here using a broken power-law cosmic ray spectra, for comparison, is seen to be lower compared to the results in [18] by up to a factor of 3. This reduction, despite the use of the same cosmic ray spectra, mainly arises due to the updated evaluation of the $Z_{p p}$ moment [as explained just below eq. (3.8)], and from differences in the large $x_{E}$ values of $d \sigma / d x_{E}$ in the dipole and perturbative QCD approaches. Figure 5b additionally shows the flux that arises for the central scale choice performed with the CTEQ3 PDFs and the broken power-law in eq. (3.7). We find that the steep increase in cross section with energy that one obtains when using this older PDF set, translates directly to larger fluxes at energies of $10 \mathrm{TeV}$ and higher (compared to central CT10 fluxes obtained using the same cosmic-ray primary flux); nonetheless, it turns out to be smaller at low energies than that obtained in the dipole computation in [18]. Finally, figure $5 \mathrm{~b}$ also shows that using scales proportional to $m_{\mathrm{c}}$ rather than $m_{\mathrm{T}}$ leads to similar central predictions but somewhat larger bands of uncertainty. This is a feature that can be traced back to the $p p \rightarrow c \bar{c} X$ cross section, as discussed in section 2 .

For both figures $5 \mathrm{a}$ and $5 \mathrm{~b}$, we show the conventional atmospheric neutrino flux from ref. [60]. Since the original computation of this flux in [60] was based on the broken powerlaw cosmic ray primary flux, for consistency, we reweigh this flux in figure 5a to account 
for the updated H3a cosmic-ray primary flux ${ }^{3}$ using the code NeutrinoFlux [61]. This reweighted flux is used also to determine the resulting conventional atmospheric background in figure 6.

Quantifying, approximately, the changes in our evaluation over that in [18] aside from using perturbative QCD rather than the dipole model approach, we note that the following factors are responsible for a reduced prompt flux:

- Use of recent cosmic-ray flux estimates which predict a lower nucleon flux at high energies $\left(10^{7}-10^{9} \mathrm{GeV}\right)$ than the previous broken power-law (see eq. (3.7)). Correspondingly, the prompt flux evaluated using the H3p cosmic ray primary flux is reduced by a factor of 1.2-3 (figure $5 \mathrm{a}$ ) relative to the broken power-law between the energies $10^{5}-10^{6} \mathrm{GeV}$, for example, where the prompt background is expected to be important.

- Reevaluation of $Z_{p p}$ and $\Lambda_{p}$, and $Z_{k k}$ and $\Lambda_{K}$, using the H3p cosmic ray flux and a parametrization of the $p \rightarrow p, D \rightarrow D \simeq K \rightarrow K$ differential cross sections, and using an updated $p$-Air cross section, decreases the flux by about $30 \%$.

- Use of CT10 PDFs instead of the older CTEQ3 PDFs used in [21] reduces the flux by an additional factor of $2-3$ at the high energies $\left(\gtrsim 10^{5} \mathrm{GeV}\right)$, as figure $5 \mathrm{~b}$ shows by comparing fluxes computed using the same broken power-law cosmic-ray primary flux and QCD parameters, but with the two different PDF sets.

- Between the dipole calculation in [18] and the results shown here for the broken power law cosmic ray flux, the large $x$ behavior of the dipole and perturbative QCD evaluations of $p A \rightarrow c \bar{c} X$, input to the evaluation of the production $Z$-moments, account for the relative fluxes differing by a factor of $\sim 1.5$. Uncertainties associated with variations of the QCD parameters for the perturbative and dipole evaluations are shown with the (overlapping) bands in figure 5b.

\section{Prompt background at IceCube}

The IceCube atmospheric neutrino background event rates, both prompt and conventional, can be evaluated from the corresponding flux by making use of the flavor-dependent effective areas for the detector, given in [15]. The central value of the estimated prompt neutrino background relevant to the 988-day IC observation is shown in figure 6. Additionally, the figure also shows the maximum and minimum estimates due to variation in the cross section (see figure 1) and differential charm quark energy distribution obtained by varying the QCD parameters compatible with experimental results. ${ }^{4}$ We note that the

\footnotetext{
${ }^{3}$ The difference between results obtained using H3a and those using H3p fluxes is negligible below 1 $\mathrm{PeV}$. Thus, we do not distinguish between the two when computing the conventional flux, which is only significant at much lower energies, i.e., up to $200 \mathrm{TeV}$.

${ }^{4}$ When comparing event rates from prompt neutrino flux obtained here to those obtained in [16], the difference is not as stark as one would expect from a straight comparison at the level of fluxes obtained here to that obtained in [18], because IC implements a reweighing procedure on the central estimate of the prompt neutrino flux in [18] to already account for updated cosmic-ray spectra.
} 


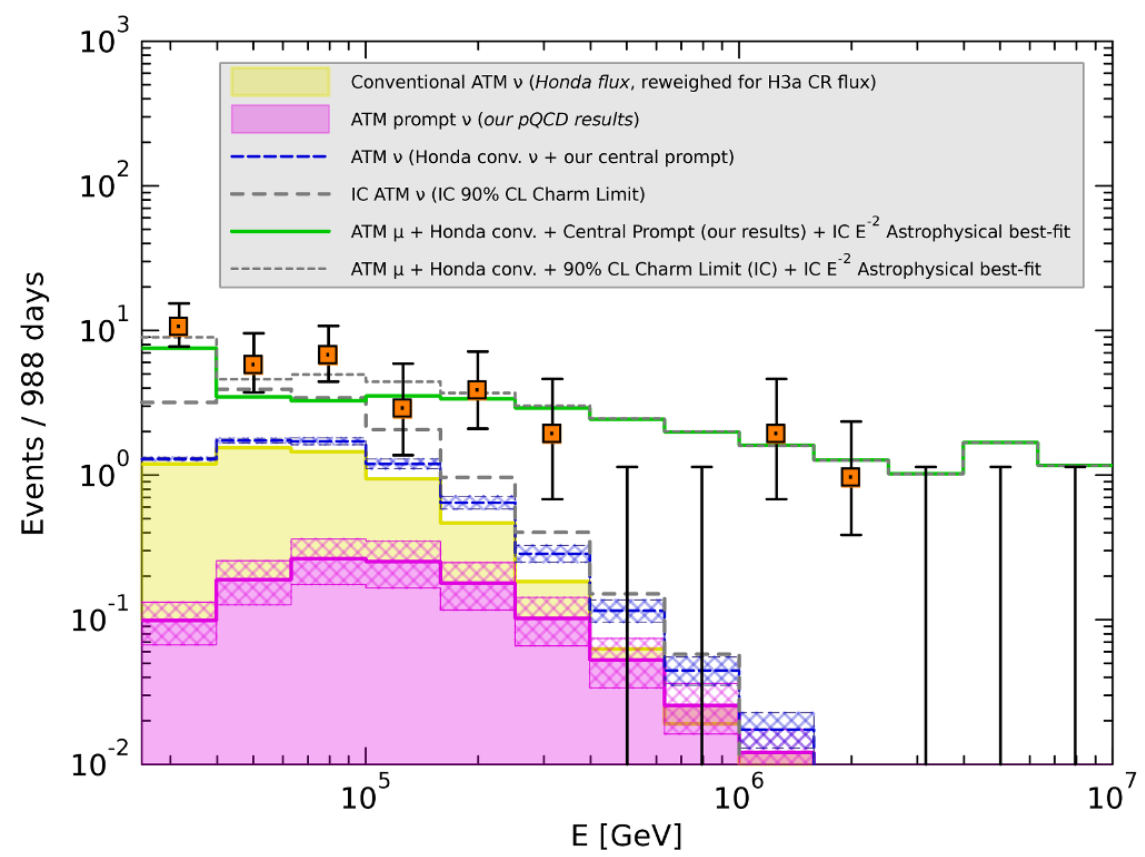

Figure 6. Event rates at IceCube from prompt neutrinos, with our updated prediction for the prompt flux indicated in magenta, along with uncertainties from variation in the QCD parameters indicated as a hatched region around the central curves. The re-evaluated total atmospheric neutrino background (blue dashed curve) includes events from our central prompt prediction and the Honda conventional neutrino flux reweighted for H3a cosmic ray flux (with the uncertainty in prompt event rates indicated as a hatched region around it). The total neutrino background estimated by IC at the level of 90\% CL charm limit [16] is shown (dashed gray curve) for comparison. The prediction for total event rates using the $E^{-2}$ fit astrophysical signal from [16] and updated atmospheric background (i.e., including our re-evaluated prompt background) is shown as a green curve, while the similar IC estimate using the older atmospheric background and prompt at the level of 90\% CL (see [16]) is shown as the gray thick-dashed curve. For both these latter curves, the background includes contribution from atmospheric muons (reproduced from [16]), in addition to atmospheric neutrinos. Observed total event rates at the IC are shown as solid red blocks, along with their associated $1 \sigma$ statistical uncertainty.

procedure using the IC effective areas overestimates the prompt event rates because it does not take into account the neutrino self-veto as discussed in [62].

Using the Honda estimates for conventional atmospheric neutrino flux, ${ }^{5}$ events from background muons estimated in [16], and our central prompt flux, we compute the total atmospheric background. This is shown in figure 6 as the blue dashed curve, with the uncertainty indicated as a hatched area around this central curve. When compared with the conventional atmospheric neutrino background, the prompt flux is only a minor contributor at energies where the background is significant (about $200 \mathrm{TeV}$ and lower). Consequently, the total atmospheric neutrino background at these energies is not significantly affected by the $\sim 10 \%-15 \%$ uncertainty in the prompt flux from variation of the QCD parameters.

\footnotetext{
${ }^{5}$ For the conventional atmospheric neutrino flux too, IC uses the Honda flux reweighted to account for changes in fits to the cosmic-ray spectra from older power-law estimates to more recent estimates, e.g. in [24]. We have used the same method in figure 5a to obtain the conventional neutrino flux.
} 
It is clear from a comparison with the total atmospheric background (gray dashed curve) in figure 6, obtained using a previous results for the prompt neutrino flux [16] and IC's experimental 90\% CL prompt flux limits, ${ }^{6}$ that our new pQCD results lead to a significant reduction in the total background in the lower energies up to $100 \mathrm{TeV}$. This will in turn lead to revisions in the evaluation of statistical significances of the astrophysical signal for the IC, but a detailed re-analysis of the IC signal is beyond the scope of the present work. We note that this reduced background implies that the IC fixed-slope $E^{-2}$ best-fit astrophysical flux underestimates the low-energy signal in the observed events.

\section{Conclusions}

Hadronic interactions at extremely high energies necessarily involves understanding of QCD at these energies; however, this is beset by relatively large uncertainties in the related computation. Interactions between cosmic ray protons and atmospheric nuclei which lead to the production of neutrinos via the formation of a mesons $\left(\pi^{ \pm}, K^{0, \pm}, D^{0, \pm}, D_{s}^{ \pm}\right)$ and baryons, and its consequent decay is one such process. In the era of the IceCube observations of ultra-high energy neutrinos, the importance of properly understanding and estimating the background from atmospheric neutrinos cannot be overstated. Since earlier perturbative QCD results for prompt neutrino fluxes were based on fairly old PDFs and were not constrained by the recent LHC data, we have revisited the computation in the present work, incorporating recent developments in the understanding of low- $x$ PDF's and using recent experimental results that have bearing on the relevant QCD inputs.

By varying the QCD parameter space including the involved factorization and renormalization scales within bounds set by results from LHCb, ALICE, ATLAS among others, and making use of recent CT10 PDF's, we have re-evaluated $\sigma(p p \rightarrow c \bar{c} X)$. We have determined $\sigma(p N \rightarrow c \bar{c} X)$ from the latter, and thereafter, using the standard procedure of energy-dependent $Z$-moments, we have computed the total prompt flux expected at the detector. For an estimate of the composition and spectrum of the cosmic ray primaries, we have relied on the recent results from ref. [24] rather than use a broken power-law which is now known to be an inaccurate representation of the cosmic ray nucleon flux at the high energies.

We find that the prompt neutrino background at the IceCube is lower than that estimated in [15]. We note that this may lead to a consequent revision in the estimates of the statistical significance of the IC signal over the now reduced atmospheric background.

\section{Acknowledgments}

We thank the IceCube collaboration for providing their code NeutrinoFlux that we used to compute the reweighted conventional neutrino fluxes, and David Boersma, Olga Botner, Teresa Montaruli and Anne Schukraft for help with the code. This research was supported

\footnotetext{
${ }^{6}$ IC's $90 \%$ CL limits on the prompt flux corresponds to 3.8 times the central prompt flux estimated in [18].
} 
in part by the US Department of Energy through contracts DE-FG02-04ER41319, DEFG02-04ER41298, DE-FG03-91ER40662, DE-FG02-13ER41976, DE-SC0010114 and DESC0002145, in part by the National Science Foundation under Grant No. NSF PHY1125915, in part by the Polish NCN grant DEC-2011/01/B/ST2/03915, and in part by the Swedish Research Council under contracts 2007-4071 and 621-2011-5107. We thank Nordita and KITP for the hospitality while part of this work was completed.

Open Access. This article is distributed under the terms of the Creative Commons Attribution License (CC-BY 4.0), which permits any use, distribution and reproduction in any medium, provided the original author(s) and source are credited.

\section{References}

[1] MACRO collaboration, S.P. Ahlen et al., Atmospheric neutrino flux measurement using upgoing muons, Phys. Lett. B 357 (1995) 481 [INSPIRE].

[2] Frejus collaboration, K. Daum et al., Determination of the atmospheric neutrino spectra with the Frejus detector, Z. Phys. C 66 (1995) 417 [InSPIRE].

[3] Super-Kamiokande collaboration, Y. Fukuda et al., Study of the atmospheric neutrino flux in the multi-GeV energy range, Phys. Lett. B 436 (1998) 33 [hep-ex/9805006] [INSPIRE].

[4] E.N. Alekseev et al., The Baksan underground scintillation telescope, Phys. Part. Nucl. 29 (1998) 254 [INSPIRE].

[5] Kamiokande collaboration, S. Hatakeyama et al., Measurement of the flux and zenith angle distribution of upward through going muons in Kamiokande II + III, Phys. Rev. Lett. 81 (1998) 2016 [hep-ex/9806038] [INSPIRE].

[6] Soudan 2 collaboration, M.C. Sanchez et al., Measurement of the L/E distributions of atmospheric neutrinos in Soudan 2 and their interpretation as neutrino oscillations, Phys. Rev. D 68 (2003) 113004 [hep-ex/0307069] [INSPIRE].

[7] MINOS collaboration, P. Adamson et al., First observations of separated atmospheric $\nu_{\mu}$ and $\bar{\nu}_{\mu}$ events in the MINOS detector, Phys. Rev. D 73 (2006) 072002 [hep-ex/0512036] [INSPIRE].

[8] MINOS collaboration, P. Adamson et al., Measurements of atmospheric neutrinos and antineutrinos in the MINOS Far Detector, Phys. Rev. D 86 (2012) 052007 [arXiv:1208.2915] [INSPIRE].

[9] T.K. Gaisser, Atmospheric neutrinos in the context of muon and neutrino radiography, Earth Planets Space 62 (2010) 195 [arXiv:0901.2386] [InSPIRE].

[10] E. Andres et al., The AMANDA neutrino telescope: Principle of operation and first results, Astropart. Phys. 13 (2000) 1 [astro-ph/9906203] [INSPIRE].

[11] F. Halzen, Astroparticle physics with high energy neutrinos: from amanda to IceCube, Eur. Phys. J. C 46 (2006) 669 [astro-ph/0602132] [INSPIRE].

[12] KM3NET collaboration, U.F. Katz, News from KM3NeT, arXiv:1403.4065 [INSPIRE].

[13] IceCube collaboration, M.G. Aartsen et al., IceCube-Gen2: A Vision for the Future of Neutrino Astronomy in Antarctica, arXiv:1412.5106 [INSPIRE]. 
[14] IceCube collaboration, R. Abbasi et al., The Energy Spectrum of Atmospheric Neutrinos between 2 and $200 \mathrm{TeV}$ with the AMANDA-II Detector, Astropart. Phys. 34 (2010) 48 [arXiv: 1004.2357] [INSPIRE].

[15] ICECube collaboration, M.G. Aartsen et al., Evidence for High-Energy Extraterrestrial Neutrinos at the IceCube Detector, Science 342 (2013) 1242856 [arXiv:1311.5238] [INSPIRE].

[16] ICECuBe collaboration, M.G. Aartsen et al., Observation of High-Energy Astrophysical Neutrinos in Three Years of IceCube Data, Phys. Rev. Lett. 113 (2014) 101101 [arXiv: 1405.5303] [INSPIRE].

[17] ICECube collaboration, M.G. Aartsen et al., Atmospheric and astrophysical neutrinos above 1 TeV interacting in IceCube, Phys. Rev. D 91 (2015) 022001 [arXiv:1410.1749] [INSPIRE].

[18] R. Enberg, M.H. Reno and I. Sarcevic, Prompt neutrino fluxes from atmospheric charm, Phys. Rev. D 78 (2008) 043005 [arXiv:0806.0418] [INSPIRE].

[19] L.V. Volkova, Energy Spectra and Angular Distributions of Atmospheric Neutrinos, Sov. J. Nucl. Phys. 31 (1980) 784 [Yad. Fiz. 31 (1980) 1510] [inSPIRE].

[20] P. Gondolo, G. Ingelman and M. Thunman, Charm production and high-energy atmospheric muon and neutrino fluxes, Astropart. Phys. 5 (1996) 309 [hep-ph/9505417] [INSPIRE].

[21] L. Pasquali, M.H. Reno and I. Sarcevic, Lepton fluxes from atmospheric charm, Phys. Rev. D 59 (1999) 034020 [hep-ph/9806428] [INSPIRE].

[22] A.D. Martin, M.G. Ryskin and A.M. Stasto, Prompt neutrinos from atmospheric $c \bar{c}$ and $b \bar{b}$ production and the gluon at very small x, Acta Phys. Polon. B 34 (2003) 3273 [hep-ph/0302140] [INSPIRE].

[23] F. Riehn, R. Engel, A. Fedynitch, T.K. Gaisser and T. Stanev, Charm production in SIBYLL, arXiv: 1502.06353 [INSPIRE].

[24] T.K. Gaisser, Spectrum of cosmic-ray nucleons, kaon production and the atmospheric muon charge ratio, Astropart. Phys. 35 (2012) 801 [INSPIRE].

[25] T.K. Gaisser, T. Stanev and S. Tilav, Cosmic Ray Energy Spectrum from Measurements of Air Showers, Front. Phys. China 8 (2013) 748 [arXiv: 1303.3565] [InSPIRE].

[26] T. Stanev, T.K. Gaisser and S. Tilav, High energy cosmic rays: sources and fluxes, Nucl. Instrum. Meth. A $\mathbf{7 4 2}$ (2014) 42 [inSPIRE].

[27] A. Bhattacharya et al., in preparation.

[28] ALICE collaboration, Measurement of charm production at central rapidity in proton-proton collisions at $\sqrt{s}=2.76 \mathrm{TeV}$, JHEP 07 (2012) 191 [arXiv: 1205.4007] [INSPIRE].

[29] ATLAS collaboration, Measurement of $D^{(*)}$ meson production cross sections in pp collisions at $\sqrt{s}=7 \mathrm{TeV}$ with the ATLAS detector, ATLAS-CONF-2011-017 (2011) [INSPIRE].

[30] LHCb collaboration, Prompt charm production in pp collisions at $\sqrt{s}=7 \mathrm{TeV}$, Nucl. Phys. B 871 (2013) 1 [arXiv:1302.2864] [INSPIRE].

[31] PHENIX collaboration, A. Adare et al., Measurement of high- $p_{\mathrm{T}}$ single electrons from heavy-flavor decays in $p+p$ collisions at $\sqrt{s}=200 \mathrm{GeV}$, Phys. Rev. Lett. 97 (2006) 252002 [hep-ex/0609010] [INSPIRE].

[32] STAR collaboration, L. Adamczyk et al., Measurements of $D^{0}$ and $D^{*}$ Production in $p+p$ Collisions at $\sqrt{s}=200 \mathrm{GeV}$, Phys. Rev. D 86 (2012) 072013 [arXiv:1204.4244] [INSPIRE]. 
[33] R.E. Nelson, R. Vogt and A.D. Frawley, Narrowing the uncertainty on the total charm cross section and its effect on the $J / \psi$ cross section, Phys. Rev. C 87 (2013) 014908 [arXiv:1210.4610] [INSPIRE].

[34] H.-L. Lai et al., New parton distributions for collider physics, Phys. Rev. D 82 (2010) 074024 [arXiv: 1007.2241] [INSPIRE].

[35] P. Nason, S. Dawson and R.K. Ellis, The Total Cross-Section for the Production of Heavy Quarks in Hadronic Collisions, Nucl. Phys. B 303 (1988) 607 [InSPIRE].

[36] P. Nason, S. Dawson and R.K. Ellis, The One Particle Inclusive Differential Cross-Section for Heavy Quark Production in Hadronic Collisions, Nucl. Phys. B 327 (1989) 49 [INSPIRE].

[37] Particle Data Group collaboration, K. Olive et al., Review of Particle Physics, Chin. Phys. C 38 (2014) 090001 [inSPIRE].

[38] M. Cacciari, M. Greco and P. Nason, The $p_{\mathrm{T}}$ spectrum in heavy flavor hadroproduction, JHEP 05 (1998) 007 [hep-ph/9803400] [INSPIRE].

[39] M. Cacciari, S. Frixione and P. Nason, The $p_{\mathrm{T}}$ spectrum in heavy flavor photoproduction, JHEP 03 (2001) 006 [hep-ph/0102134] [INSPIRE].

[40] M.L. Mangano, P. Nason and G. Ridolfi, Heavy quark correlations in hadron collisions at next-to-leading order, Nucl. Phys. B 373 (1992) 295 [INSPIRE].

[41] H.L. Lai et al., Global QCD analysis and the CTEQ parton distributions, Phys. Rev. D 51 (1995) 4763 [hep-ph/9410404] [INSPIRE].

[42] A.D. Martin, W.J. Stirling, R.S. Thorne and G. Watt, Parton distributions for the LHC, Eur. Phys. J. C 63 (2009) 189 [arXiv:0901.0002] [INSPIRE].

[43] HERA-B collaboration, I. Abt et al., Measurement of $D^{0}, D^{+}, D_{s}^{+}$and $D^{*+}$ Production in Fixed Target $920 \mathrm{GeV}$ Proton-Nucleus Collisions, Eur. Phys. J. C 52 (2007) 531 [arXiv: 0708.1443] [INSPIRE].

[44] C. Lourenco and H.K. Wohri, Heavy flavour hadro-production from fixed-target to collider energies, Phys. Rept. 433 (2006) 127 [hep-ph/0609101] [INSPIRE].

[45] J. Engel, T.K. Gaisser, T. Stanev and P. Lipari, Nucleus-nucleus collisions and interpretation of cosmic ray cascades, Phys. Rev. D 46 (1992) 5013 [INSPIRE].

[46] E789 collaboration, M.J. Leitch et al., Nuclear dependence of neutral D meson production by $800 \mathrm{GeV} / \mathrm{c}$ protons, Phys. Rev. Lett. 72 (1994) 2542 [INSPIRE].

[47] SELEX collaboration, A. Blanco-Covarrubias et al., Nuclear Dependence of Charm Production, Eur. Phys. J. C 64 (2009) 637 [arXiv:0902.0355] [INSPIRE].

[48] B.A. Kniehl and G. Kramer, Charmed-hadron fragmentation functions from CERN LEP1 revisited, Phys. Rev. D 74 (2006) 037502 [hep-ph/0607306] [INSPIRE].

[49] T.K. Gaisser, Cosmic rays and particle physics, Cambridge University Press (1990).

[50] P. Lipari, Lepton spectra in the earth's atmosphere, Astropart. Phys. 1 (1993) 195 [InSPIRE].

[51] T. Pierog and K. Werner, EPOS Model and Ultra High Energy Cosmic Rays, Nucl. Phys. Proc. Suppl. 196 (2009) 102 [arXiv:0905.1198] [InSPIRE].

[52] Pierre Auger collaboration, P. Abreu et al., Measurement of the proton-air cross-section at $\sqrt{s}=57 \mathrm{TeV}$ with the Pierre Auger Observatory, Phys. Rev. Lett. 109 (2012) 062002 [arXiv: 1208.1520] [INSPIRE]. 
[53] L. Pasquali and M.H. Reno, Tau-neutrino fluxes from atmospheric charm, Phys. Rev. D 59 (1999) 093003 [hep-ph/9811268] [INSPIRE].

[54] A.D. Panov et al., Energy Spectra of Abundant Nuclei of Primary Cosmic Rays from the Data of ATIC-2 Experiment: Final Results, Bull. Russ. Acad. Sci. Phys. 73 (2009) 564 [arXiv:1101.3246] [INSPIRE].

[55] H.S. Ahn et al., Energy spectra of cosmic-ray nuclei at high energies, Astrophys. J. 707 (2009) 593 [arXiv:0911.1889] [INSPIRE].

[56] H.S. Ahn et al., Discrepant hardening observed in cosmic-ray elemental spectra, Astrophys. J. 714 (2010) L89 [arXiv: 1004.1123] [INSPIRE].

[57] PAMELA collaboration, O. Adriani et al., PAMELA Measurements of Cosmic-ray Proton and Helium Spectra, Science 332 (2011) 69 [arXiv:1103.4055] [INSPIRE].

[58] E.V. Bugaev, A. Misaki, V.A. Naumov, T.S. Sinegovskaya, S.I. Sinegovsky and N. Takahashi, Atmospheric muon flux at sea level, underground and underwater, Phys. Rev. D 58 (1998) 054001 [hep-ph/9803488] [INSPIRE].

[59] T. Sjöstrand, High-energy physics event generation with PYTHIA 5.7 and JETSET 7.4, Comput. Phys. Commun. 82 (1994) 74 [INSPIRE].

[60] M. Honda, T. Kajita, K. Kasahara, S. Midorikawa and T. Sanuki, Calculation of atmospheric neutrino flux using the interaction model calibrated with atmospheric muon data, Phys. Rev. D 75 (2007) 043006 [astro-ph/0611418] [INSPIRE].

[61] ICECuBE collaboration, personal communication.

[62] T.K. Gaisser, K. Jero, A. Karle and J. van Santen, Generalized self-veto probability for atmospheric neutrinos, Phys. Rev. D 90 (2014) 023009 [arXiv: 1405.0525] [InSPIRE]. 